\title{
Pain Outcomes of Inpatient Pain and Palliative Care Consultations: Differences by Race and Diagnosis
}

\author{
Christina L. Bell, M.D., M.Sc., Meiko Kuriya, M.D., ${ }^{2}$ and Daniel Fischberg, M.D., Ph.D.,3
}

\begin{abstract}
Background: Pain management disparities exist among patients not receiving palliative care. We examined pain outcomes for disparities among patients receiving palliative care.

Methods: At a 542-bed teaching hospital in Honolulu, The Queens' Medical Center Pain and Palliative Care Department collected patient characteristics and pain severity (initial, final) for each consultation from 2005 through 2009. Analyses compared pain levels by race (white, Asian, Hawaiian/Pacific Islander [PI], other) and consultation diagnosis (cancer, noncancer medical, surgical [59\% orthopedic], other). Multiple regression models analyzed factors associated with lower final pain levels and pain reduction.

Results: Study population included 4658 patients. No final pain was reported by more non-white patients $(33 \%-$ $39 \%)$ than white $(27 \%, p<0.0001)$ and more cancer and noncancer medical patients $(45 \%-54 \%)$ than surgical/ other patients $(20 \%-31 \%, p<0.0001)$. Asian (adjusted odds ratio [aOR] 1.24; 95\% confidence interval [CI] $1.06-$ $1.46 ; p=0.007$ ) and PI (aOR 1.46, 95\% CI 1.20-1.77, $p=0.0001$ ) races had increased likelihoods of lower final pain severity versus whites, controlling for age, gender, Karnofsky score, preconsult length of stay, and initial pain severity. Surgical diagnoses had decreased likelihood of lower final pain levels versus cancer (aOR $0.38,95 \%$ CI $0.32-0.46, p<0.0001)$. Among 2304 patients reporting moderate/severe initial pain, $1738(75.4 \%)$ reported pain reduction to mild/no final pain. PI race was associated with pain reduction versus whites (aOR 1.57, 95\% CI $1.17-2.10, p=0.003)$. Surgical diagnoses had decreased likelihood of pain reduction vs. cancer (aOR $0.52,95 \% \mathrm{CI}$ $0.39-0.71, p<0.0001)$.
\end{abstract}

Conclusion: Pain outcomes were similar or better among non-white races than whites. Surgical patients reported more final pain than cancer patients.

\section{Introduction}

$\mathbf{T}$ HE EXAMINATION OF pain management across diverse populations is an important area of research in palliative medicine. ${ }^{1-3}$ Recent studies describe disparities in the treatment of pain for patients with cancer ${ }^{4}$ and patients in emergency departments. ${ }^{5}$

Palliative care, with its focus on pain and symptom management, effective communication skills and equitable access to care for all patients, ${ }^{6}$ may be able to overcome obstacles to pain management among non-white patients. Data are lacking on whether patients who receive palliative care consultations also demonstrate these racial disparities in pain management.

Reports on pain management outcomes from inpatient hospital palliative care consultations have been limited. ${ }^{7}$ It is important to examine pain outcomes from an inpatient palliative care service, since in many communities, the hospital setting is the only place patients can access palliative care without enrolling in a hospice program. Studies have indicated that non-white patients may have lower rates of hospice use than white patients. ${ }^{8,9}$ Thus, inpatient palliative care consultation services may care for a more diverse patient population than hospice programs. Previous studies including Asian American and Pacific Islander populations receiving consultations for pain or palliative care did not compare pain outcomes by racial categories. ${ }^{10-12}$ No study has examined pain management by an inpatient hospital-based palliative care consultation service among different races and diagnoses.

It is important to examine pain management by patient characteristics to improve the quality of care provided for all patients. Studying the patterns of pain outcomes by diagnoses

\footnotetext{
${ }^{1}$ Department of Geriatric Medicine, John A. Burns School of Medicine, University of Hawaii, Honolulu, Hawaii.

${ }^{2}$ Department of Palliative and Supportive Care, Seirei Mikatahara General Hospital, Shizuoka, Japan.

${ }^{3}$ Pain and Palliative Care Department, The Queen's Medical Center, Honolulu, Hawaii.

Accepted June 7, 2011.
} 
of patients broadens the understanding of palliative care challenges and opportunities in noncancer center settings. This study examined the impact of a palliative care consultation on pain outcomes using data collected prospectively at a major community-based referral hospital serving a diverse patient population. The study specifically examined pain outcomes after consultation by patient race and consultation diagnosis in order to: (1) identify possible racial disparities in pain outcomes among patients receiving palliative care consultations and (2) identify patient groups who currently benefit from the consultations and patient groups who may need additional intervention or study to enhance their pain management.

\section{Methods}

\section{Participants, design, and setting}

The Queen's Medical Center, a 542-bed community-based teaching hospital, is the major tertiary care referral center for the Pacific basin. The diverse patient population reflects the general population of Hawaii and the Pacific. In 2004, the nurse-run Pain Management Service converted to an interdisciplinary Pain \& Palliative Care Department developed to meet the needs of hospitalized patients with acute pain, acute exacerbations of chronic pain, or palliative care needs including pain related to progressive or life-limiting illness. This study consisted of an analysis of data from the Pain and Palliative Care Department at The Queen's Medical Center. All adult hospital patients, with a recorded consultation diagnosis, at least 1 day of inpatient hospital stay and who received inpatient pain and palliative care team consultations from January 1, 2005 through December 31, 2009 were included. This study was approved by the University of Hawaii and The Queen's Medical Center Institutional Review Boards. Requirement for informed consent was waived based on the retrospective analyses of a de-identified dataset that was prospectively compiled during usual patient care over 5 years, making individual patient consent unfeasible.

\section{Instrument procedures}

Patient demographic characteristics, Karnofsky score, and consultation diagnosis were recorded at the time of the consultation. Pain scores were prospectively obtained for each patient at the time of consultation and prior to discharge. Scores were based on patient report of pain at that moment using an 11-point numeric rating (0-10) scale. A verbal rating (none, mild, moderate, severe) was used when patients preferred to use the conceptually simple $\mathrm{e}^{13}$ adjective rating instead of a numeric pain score using the 11-point scale. ${ }^{14}$ The verbal rating scale is often preferred by older patients or those with difficulty with abstract questions. ${ }^{13,15,16}$ For analyses, in order to include the broadest population possible (including elderly patients and those unable to use the numeric scale), the 11-point numeric scale ratings were collapsed to the 4-point scale as follows: 0 , no pain (rank $=0) ; 1$ to 3 , mild (rank $=1) ; 4$ to 6 , moderate (rank $=2$ ); and 7 to 10 , severe $($ rank $=3) .{ }^{17,18}$ In a previous study comparing the verbal rating scale with 11point scales, a cut point of 7 and higher to distinguish severe from nonsevere pain had a sensitivity of $89.6 \%$ and specificity of $79.6 \%$. A cut point of 4 and higher to distinguish moderate to severe pain from mild or no pain had a sensitivity of $90.3 \%$ and a specificity of $79.2 \% .{ }^{17}$ Scores were primarily based on patient report; surrogate report was used when patient report was not possible. Clinicians observed the level of pain behaviors (bracing, grimacing, restlessness, rubbing, and vocalizing) based on the Checklist of Nonverbal Pain Indicators ${ }^{19}$ and graded pain as absent, mild, moderate, or severe only when neither patient nor surrogate report was possible. For a sample year with available pain score source data, $86 \%$ of pain scores were patient-generated, $13 \%$ were clinician-generated and $1 \%$ was surrogate-generated.

\section{Definitions}

Racial data were obtained from patient self-report at the time of hospital admission. Races ${ }^{20}$ reflected Hawaii's diverse population and consisted of Caucasian, Japanese, Okinawan, Hawaiian, part-Hawaiian, Korean, Chinese, Micronesian, Pacific Islander, Filipino, African American, Native American, Middle Eastern, East Indian, and mixed races. For analyses, the racial categories were clustered into the four largest groups: Caucasian or white; Asian; Hawaiian, part-Hawaiian, Pacific Islander and Micronesian; and other races. ${ }^{21}$

Diagnosis data reflected the palliative care clinician's assessment at the time of the initial palliative care evaluation of the major diagnosis leading to the consultation. This was a clinical assessment taking into account the medical record, the patient's history and findings on physical examination. Billing ICD-9 codes were not used to determine the diagnosis. The diagnoses were sorted into general categories including cancer and the following noncancer diagnoses: orthopedic, trauma, general surgical, urologic, gynecologic, cardiac, hepatic, stroke/coma, renal, pulmonary, dementia, psychiatric and other diagnoses not listed. For analyses, the diagnoses were grouped according to type of care the patient received in the hospital: surgical care (including orthopedic, trauma, general surgical, urologic and gynecologic), oncologic care, noncancer medical care (including cardiac, hepatic, stroke/coma, renal, pulmonary and dementia), or psychiatric/other/unknown, based on this primary diagnosis. These groupings were necessary to examine any patterns in pain management between different types of hospital patients receiving palliative care consultation. The consulting pain and palliative care team estimated the Karnofsky score at the initial consultation in 10\% increments (10-100), as a proxy for overall level of illness. ${ }^{22}$ Preconsultation hospital length of stay was recorded in days.

\section{Outcomes}

The main outcome of interest was level of final pain severity as an ordinal variable. Pain was also analyzed as reduction in pain from moderate or severe to mild or none, excluding patients with mild or no initial pain. For pain reduction analyses, a dichotomous (yes/no) pain reduction variable was created. Patients with reduction in pain from moderate or severe (initial) to mild or none (final) were assigned a "yes" value (1) for pain reduction. Patients with final pain levels of moderate or severe were assigned a "no" value (0) for pain reduction.

\section{Analyses}

Descriptive statistics of the patient population were generated, and characteristics of patients were compared using $\chi^{2}$ and analysis of variance (ANOVA). The 4-point verbal rating 
scale is a rank of pain, and the intervals between the ranks cannot be assumed to be equal. The Kruskal-Wallis statistic (an ordinal measure test analogous to the $t$ test or ANOVA for continuous measures) was used to determine significance of difference in levels of initial and final pain severity by patient race and diagnosis. The pain outcomes were analyzed in multiple regression models in two different ways. First, factors associated with lower severity of final pain levels versus higher levels were analyzed using ordinal logistic regression models, controlling for age, race, diagnosis, gender, Karnofsky score, preconsult length of stay, and initial pain severity. A subgroup analysis excluded patients with no or mild initial pain, as patients with moderate or severe initial pain at the time of the first palliative care evaluation were most in need of effective pain management. Second, a multiple logistic regression model examined factors associated with reduction in final pain severity to none or mild among patients with moderate or severe pain on initial pain and palliative care evaluation, adjusting for the same covariates as the ordinal logistic regression model.

\section{Results}

Demographic and clinical characteristics are displayed in Table 1. A total of 4658 adult patients received pain and pal- liative care consultations during the five calendar years 2005 through 2009 . Over $60 \%$ of the patients who received consultation were aged less than 65 years and 51\% were male. Over $25 \%$ of patients had cancer as the primary diagnosis resulting in the consultation, while $46 \%$ had surgical diagnoses. The median Karnofsky score was $60 \%$. The mean number of hospital days prior to consultation was 5.8 days. While initial pain was severe in $29 \%$ of patients and moderate in $35 \%$, final pain was severe in $2 \%$ and moderate in $16 \%$. Among the 2304 patients with moderate or severe pain initially, 75\% had final pain reduced to mild or none.

Characteristics by consultation diagnosis are displayed in Table 2. The mean Karnofsky score was higher in the surgical $(70 \%)$ and "other" diagnoses (60\%) compared to the cancer $(40 \%)$ and noncancer medical diagnoses (48\%). The percentage of patients with severe initial pain was similar (26\%-29\%) across diagnoses, although the "other diagnoses" group had the highest percentage $(34 \%)$ of patients with severe initial pain. A higher percentage of patients with cancer and noncancer medical diagnoses (21\% and 23\%, respectively) reported no initial pain compared to surgical (8.1\%) and other diagnoses $(7.8 \%)$. Less than $5 \%$ of patients in any diagnosis group reported severe final pain, but more patients in the surgical and "other" diagnoses groups reported moderate final pain (20\% and $16 \%$, respectively). Significantly more

Table 1. Characteristics of Patients by Racial Category

\begin{tabular}{|c|c|c|c|c|c|c|}
\hline Characteristic & $\begin{array}{c}\text { Overall } \\
\mathrm{n}=4658(\%)\end{array}$ & $\begin{array}{c}\text { White } \\
\mathrm{n}=2022(43.4 \%)\end{array}$ & $\begin{array}{c}\text { Asian } \\
\mathrm{n}=1601(34.4 \%)\end{array}$ & $\begin{array}{c}\text { Hawaiian/Islander } \\
\mathrm{n}=820(17.6 \%)\end{array}$ & $\begin{array}{c}\text { Other race } e^{\mathrm{a}} \\
\mathrm{n}=215(4.6 \%)\end{array}$ & $\mathrm{p}$ value \\
\hline Age $<65$ years & $2955(63.5)$ & $1369\left(67.7^{\mathrm{b}}\right)$ & $780(48.8)$ & $651(79.4)$ & $155(72.1)$ & $<0.0001$ \\
\hline Age $65-74$ years & 822 (17.7) & $344(17.0)$ & $318(19.9)$ & $125(15.2)$ & $35(16.3)$ & \\
\hline Age 75 years and older & $880(18.9)$ & 309 (15.3) & $502(31.4)$ & $44(5.4)$ & 25 (11.6) & \\
\hline Male gender & $2390(51.3)$ & 1042 (51.6) & $781(48.8)$ & $442(53.9)$ & $125(58.1)$ & 0.02 \\
\hline Female gender & $2267(48.7)$ & $979(48.4)$ & $820(51.2)$ & $378(46.1)$ & $90(41.9)$ & \\
\hline \multicolumn{7}{|l|}{ Consultation diagnosis } \\
\hline Cancer & 1255 (25.9) & 365 (18.1) & $560(35.0)$ & $275(33.5)$ & $55(25.6)$ & $<0.0001$ \\
\hline Surgical $^{\mathrm{C}}$ & $2163(46.4)$ & $1093(54.1)$ & $677(42.3)$ & $295(36.0)$ & $98(45.6)$ & \\
\hline Noncancer Medical $^{\mathrm{d}}$ & $528(11.3)$ & $218(10.8)$ & $160(10.0)$ & $122(14.9)$ & $28(13.0)$ & \\
\hline Other Diagnosis $^{\mathrm{e}}$ & $712(15.3)$ & $346(17.1)$ & $204(12.7)$ & $128(15.6)$ & $34(15.8)$ & \\
\hline Mean Karnofsky score & $58.0 \pm 25.3$ & $61.8 \pm 23.6$ & $54.8 \pm 26.6$ & $54.3 \pm 25.2$ & $60.4 \pm 25.3$ & $<0.0001$ \\
\hline Preconsult $\operatorname{LOS}^{f}$ & $5.8 \pm 16.5$ & $4.7 \pm 12.4$ & $6.7 \pm 21.4$ & $7.2 \pm 15.4$ & $4.8 \pm 10.4$ & $<0.0001$ \\
\hline \multicolumn{7}{|l|}{ Initial pain severity } \\
\hline None & $584(13.3)$ & $158(8.2)$ & $294(19.8)$ & $103(13.3)$ & $29(14.2)$ & $<0.0001$ \\
\hline Mild & 987 (22.5) & $429(22.4)$ & $376(25.3)$ & $142(18.3)$ & 40 (19.6) & \\
\hline Moderate & 1529 (34.9) & $708(36.9)$ & 459 (30.9) & $289(37.2)$ & $73(35.8)$ & \\
\hline Severe & $1282(29.3)$ & $622(32.5)$ & 355 (23.9) & $243(31.3)$ & $62(30.4)$ & \\
\hline \multicolumn{7}{|l|}{ Final pain severity } \\
\hline None & $1216(33.0)$ & $439(26.5)$ & $480(39.0)$ & $243(38.5)$ & $54(32.5)$ & $<0.0001$ \\
\hline Mild & $1808(49.0)$ & $845(51.0)$ & $590(47.9)$ & $296(46.8)$ & 77 (46.4) & \\
\hline Moderate & 574 (15.6) & $320(19.3)$ & 139 (11.3) & $83(13.1)$ & 32 (19.3) & \\
\hline Severe & $90(2.4)$ & $54(3.3)$ & $23(1.9)$ & $10(1.6)$ & $3(1.8)$ & \\
\hline Pain reduced ${ }^{g}$ & $1738(75.4)$ & $796(71.5)$ & $518(78.7)$ & $349(81.4)$ & $75(72.8)$ & $<0.0001$ \\
\hline
\end{tabular}

a'Other race group: African American $(n=60,1.3 \%)$, other races (other American/Native, cosmopolitan, unknown, Middle Eastern, declined, $n=39,0.7 \%)$, and missing race $(n=116,2.6 \%$, including patients who reported Hispanic ethnicity but did not report race $(n=90$, $2.0 \%$.

${ }^{\mathrm{b}}$ Column percentages shown for racial groups.

'Surgical group: orthopedic $(n=1277,27.4 \%)$, trauma $(n=268,5.8 \%)$, surgery $(n=575,12.3 \%)$, gynecologic $(n=19, n=0.4 \%)$ and urologic $(n=24,0.5 \%)$.

${ }^{\mathrm{d}}$ Noncancer medical diagnoses included heart $(n=224,4.8 \%)$, liver $(n=29,0.6 \%)$, stroke/coma $(n=30,0.6 \%)$, kidney $(n=49,1.1 \%), 1$ lung $(n=152,3.3 \%)$, dementia $(n=14,0.3 \%)$, diabetes $(n=12,0.3 \%)$, and HIV/AIDS $(n=18,0.4 \%)$.

e Other diagnoses included psychiatric $(n=16,0.3 \%)$ and other diagnoses not listed $(n=696,14.9 \%)$.

${ }^{\mathrm{f}}$ Length of stay (LOS) in the hospital prior to palliative care consultation, in mean number of days.

gPain reduced among 2304 patients with initial pain moderate or severe, to final pain mild or moderate. 
Table 2. Patient Characteristics and Pain Levels by Primary Diagnosis Requiring Consultation

\begin{tabular}{|c|c|c|c|c|c|}
\hline Characteristic & $\begin{array}{c}\text { Cancer } \\
\mathrm{n}=1255(25.9 \%)\end{array}$ & $\begin{array}{c}\text { Surgical } \\
\mathrm{n}=2163(46.5 \%)\end{array}$ & $\begin{array}{c}\text { Noncancer medical } \\
\mathrm{n}=528(11.3 \%)\end{array}$ & $\begin{array}{l}\text { Other diagnosis } \\
\mathrm{n}=712(15.3 \%)\end{array}$ & $\mathrm{p}$ value \\
\hline Age $<65$ years & $770(61.4)$ & $1429(66.1)$ & $291(55.1)$ & $465(65.3)$ & $<0.0001$ \\
\hline Age $65-74$ years & $259(20.7)$ & 354 (16.4) & $108(20.5)$ & $101(14.2)$ & \\
\hline Age 75 years and older & $225(17.9)$ & $380(17.6)$ & $129(24.4)$ & $146(20.5)$ & \\
\hline Male gender & $643(51.2)$ & $1095(50.6)$ & $318(60.3)$ & $334(46.9)$ & $<0.0001$ \\
\hline Female gender & $612(48.8)$ & $1068(49.4)$ & 209 (39.7) & $378(53.1)$ & \\
\hline Karnofsky score (mean) & $40.9 \pm 20.6$ & $69.8 \pm 20.2$ & $48.2 \pm 27.3$ & $60.7 \pm 24.8$ & $<0.00001$ \\
\hline Mean preconsult LOS & $7.2 \pm 16.4$ & $3.6 \pm 14.8$ & $10.5 \pm 19.6$ & $6.8 \pm 17.8$ & $<0.00001$ \\
\hline \multicolumn{6}{|l|}{ Initial pain severity } \\
\hline None & $252(21.0)$ & $164(8.1)$ & $116(23.0)$ & $52(7.8)$ & $<0.0001$ \\
\hline Mild & $284(23.7)$ & $470(23.3)$ & $108(21.4)$ & $125(18.8)$ & \\
\hline Moderate & $327(27.3)$ & 795 (39.5) & $146(29.0)$ & $261(39.3)$ & \\
\hline Severe & $335(28.0)$ & $586(29.1)$ & $134(26.6)$ & $227(34.1)$ & \\
\hline \multicolumn{6}{|l|}{ Final pain severity } \\
\hline None & $502(54.0)$ & $372(20.4)$ & $167(44.8)$ & $175(31.4)$ & $<0.0001$ \\
\hline Mild & $334(35.9)$ & $1043(57.1)$ & $160(42.9)$ & $271(48.6)$ & \\
\hline Moderate & $82(8.8)$ & 361 (19.8) & 42 (11.3) & 89 (16.0) & \\
\hline Severe & 12 (1.3) & $51(2.8)$ & $4(1.1)$ & $23(4.1)$ & \\
\hline Pain reduced ${ }^{\mathrm{a}}$ & $434(84.8)$ & 839 (70.3) & $172(81.1)$ & $293(75.9)$ & $<0.0001$ \\
\hline
\end{tabular}

aPain reduced among patients with initial pain severity of moderate or severe, to final pain severity of mild or moderate, $n=$ For each additional day the likelihood of a lower pain level increased 1\%.

patients with cancer and noncancer medical diagnoses reported no final pain ( $54 \%$ and $45 \%$, respectively), than patients with surgical (20\%) and other (31\%) diagnoses. Eightyfive percent of cancer and $81 \%$ of noncancer medical patients had clinically significant pain reduction from severe or moderate to mild or none, compared to patients with surgical (70\%) and other $(76 \%)$ diagnoses.

Lower levels of initial pain were associated with several factors in adjusted analyses. Increased likelihoods of lower levels of initial pain were significantly associated with older age (OR 2.29, 95\% CI 1.97-2.65, $p<0.0001$, data not shown), Asian race (OR 1.50, 95\% CI 1.31-1.70, p <.0001, versus white race, data not shown), and preconsult length of stay (OR 1.01, 95\% CI 1.01-1.01, data not shown). Decreased likelihood of lower levels of initial pain were significantly associated with surgical (OR 0.70, 95\% CI 0.60-0.82, $p<0.0001$, data not shown) and other diagnoses (OR 0.55, 95\% CI 0.46-0.66, $p<0.0001$, data not shown). There were no significant differences in association with lower levels of initial pain between Hawaiian/Pacific Islander ethnicity, "other" ethnicity and white ethnicity (data not shown).

Factors associated with lower levels of final pain severity, controlling for gender, Karnofsky score, preconsult length of stay and initial pain severity are displayed in Table 3. Age older than 75 years was associated with increased adjusted likelihood of lower final pain levels compared to patients younger than 75 years. Asian and Hawaiian/Pacific Islander races were associated with increased adjusted likelihoods of

Table 3. Factors Associated with Likelihood of Lower Pain Severity Ranking at Discharge: Multiple Ordinal Logistic Regression

\begin{tabular}{|c|c|c|c|c|}
\hline Factor & OR $(95 \% C I)^{\mathrm{a}}$ & $\mathrm{p}$ value & OR $(95 \% C I)^{\mathrm{b}}$ & $\mathrm{p}$ value \\
\hline 75 and older (vs. <75) & 1.43 (1.19-1.72) & 0.0002 & $1.72(1.34-2.20)$ & $<0.0001$ \\
\hline Male (vs. female) & $1.00(0.87-1.14)$ & 0.9 & $1.01(0.86-1.19)$ & 0.9 \\
\hline White (ref) & 1.00 & & 1.00 & \\
\hline Asian & $1.24(1.06-1.46)$ & 0.007 & $1.12(0.92-1.36)$ & 0.3 \\
\hline Hawaiian Pacific Islander & $1.46(1.20-1.77)$ & 0.0001 & $1.44(1.15-1.80)$ & 0.002 \\
\hline Other race & 1.05 (0.76-1.47) & 0.8 & 0.99 (0.67-1.47) & 0.9 \\
\hline Cancer (ref) & 1.00 & & 1.00 & \\
\hline Surgical & $0.38(0.32-0.46)$ & $<0.0001$ & $0.42(0.33-0.52)$ & $<0.0001$ \\
\hline Noncancer medical & $0.73(0.56-0.94)$ & 0.02 & $0.69(0.50-0.96)$ & 0.03 \\
\hline Other diagnosis & $0.54(0.43-0.68)$ & $<0.0001$ & $0.56(0.43-0.74)$ & $<0.0001$ \\
\hline Karnofsky score ${ }^{c}$ & $0.99(0.99-0.99)$ & $<0.0001$ & $0.99(0.99-0.99)$ & $<0.0001$ \\
\hline Preconsult LOS ${ }^{\mathrm{d}}$ & $1.01(1.00-1.01)$ & 0.01 & $1.01(1.00-1.01)$ & 0.05 \\
\hline Initial pain level & $0.51(0.47-0.55)$ & $<0.0001$ & $0.64(0.54-0.75)$ & $<0.0001$ \\
\hline
\end{tabular}

${ }^{a} \mathrm{OR}=$ odds ratio; $95 \% \mathrm{CI}=95 \%$ confidence interval. OR is the likelihood of having a lower level of pain vs. higher levels.

${ }^{\mathrm{b}}$ Subgroup analysis excluding patients with no pain (0) or mild pain (1) initially.

${ }^{\mathrm{c}}$ For every $10 \%$ increase in Karnofsky score the likelihood of a lower pain level decreased $1 \%$.

${ }^{\mathrm{d}}$ For each additional day the likelihood of a lower pain level increased $1 \%$. 
Table 4. Factors Associated with Reduction in Pain from Moderate or Severe (Initial Consult Visit) to None or Mild (Final Consult Visit): Multiple Logistic Regression

\begin{tabular}{lcc}
\hline Factor & OR $(95 \%$ CI $)$ & $\mathrm{p}$ value \\
\hline 75 and older (vs. <75) & $2.27(1.57-3.27)$ & $<0.0001$ \\
Male (vs. female) & $0.93(0.76-1.13)$ & 0.5 \\
White (ref) & 1.00 & \\
Asian & $1.19(0.94-1.52)$ & 0.2 \\
Hawaiian Pacific Islander & $1.57(1.17-2.10)$ & 0.003 \\
Other race & $1.01(0.63-1.60)$ & 0.9 \\
Cancer (ref) & 1.00 & \\
Surgical & $0.52(0.39-0.71)$ & $<0.0001$ \\
Noncancer Medical & $0.84(0.54-1.30)$ & 0.4 \\
Other diagnosis & $0.65(0.45-0.94)$ & 0.02 \\
Karnofsky score & $0.99(0.99-1.00)$ & 0.02 \\
Preconsult LOS & $1.01(1.00-1.02)$ & 0.2 \\
Initial Pain level & $0.62(0.50-0.75)$ & $<0.0001$ \\
\hline
\end{tabular}

LOS, length of stay.

lower final pain levels than white race, although Asian race was no longer significant when patients with mild or no initial pain were excluded. Surgical, noncancer medical, and other diagnoses were associated with decreased adjusted likelihoods of lower pain levels at discharge compared to cancer diagnoses.

Factors associated with reduction in pain severity to mild or none from moderate or severe are displayed on Table 4. This analysis excluded patients with mild or no initial pain $(n=2304)$. Controlling for the same covariates, an increased adjusted likelihood of reduction in pain severity was associated with age older than 75 years compared to those younger than 75 years and Hawaiian/Pacific Islander race compared to whites. Surgical and other diagnoses were associated with a decreased adjusted likelihood of reduction in pain severity compared to cancer. Results on Tables 3 and 4 did not significantly change after excluding patients who died during the hospitalization.

\section{Discussion}

This is the first study, to the authors' knowledge, to examine pain outcomes of inpatient palliative care consultations by patient race and diagnosis. This study did not find evidence of racial disparities in pain outcomes among non-white patients receiving inpatient palliative care consultations compared to white patients. However, the study clearly indicated associations between race, diagnosis, and pain outcomes of palliative care consultations. Compared to white patients, Hawaiian and Pacific Islander patients were $46 \%$ more likely to have a lower final pain severity than white patients. Compared to patients with cancer, patients with surgical pain were over $60 \%$ less likely to have lower final pain severity and $48 \%$ less likely to have pain reduction from moderate or severe to mild or none.

Overall, $75 \%$ of all patients with moderate or severe pain had clinically significant reduction in pain to mild or none. Interestingly, older patients with moderate to severe initial pain were over twice as likely to have pain reduction as younger patients. Better function on Karnofsky score was associated with reduced likelihood of lower final pain sever- ity. Longer hospital stay prior to consultation was associated with lower final pain severity.

The racial differences in pain outcomes are in contrast to studies describing significant disparities in pain management. ${ }^{2,3,5,23-25}$ Previous studies demonstrating disparities did not examine patients receiving palliative care consultations. The non-white races included in this study are predominantly Asian and Pacific Islander, are different from most previous research samples, and have received little previous study.

Previous studies have demonstrated variation in pain measurement and reporting among races. ${ }^{26,27}$ In some studies, non-white patients reported higher levels of pain than white patients. ${ }^{28,29}$ Racial variation in pain reporting may particularly be an issue ${ }^{30}$ when pain is measured with affective terms. ${ }^{26}$ However, in this study, a lower percentage of Asian race patients reported severe and moderate pain compared to patients of the other races. There were no significant differences between Hawaiian or Pacific Islander race, other races and white race in likelihood of lower initial pain levels, even after adjusting for diagnoses. Thus, the superior pain outcomes among Hawaiian and Pacific Islander race patients compared to white patients were unlikely to be due to underreporting of pain or lower levels of initial pain.

Pain management variation, noted in other studies, ${ }^{5,31}$ is an unlikely explanation for the final pain severity variation in this study. Standard pain management guidelines ${ }^{32-34}$ were used for the initiation and titration of all pain medications. Similarly, racial variations in opioid sensitivity ${ }^{35}$ are unlikely to have affected pain reduction in this study. The patients with moderate or severe pain were referred for consultation when they could not be adequately managed by the primary team. At least $71 \%$ of patients in all the racial groups reported pain reduction to mild or no pain after consultation.

This study highlights some potential pain management challenges for inpatient palliative care teams, particularly patients with noncancer surgical diagnoses. More non-white patients had cancer diagnoses, while more white patients had surgical, particularly orthopedic, diagnoses, often with longstanding chronic pain issues. Thus, a subset of the patients (predominantly white with chronic orthopedic pain) may represent a more refractory pain management population than may be seen in many cancer centers, yet may be common in community hospital settings. However, even after excluding white patients with orthopedic diagnoses, the magnitude and significance of the likelihood ratios for pain outcomes associated with race and diagnosis persisted (data not shown).

There have been few previous studies comparing palliative care team pain management among patients with both cancer and noncancer diagnoses. ${ }^{36,37}$ Much of the palliative care literature focuses on cancer pain. ${ }^{38,39}$ The hospital in this study does not have an anesthesia-based pain management service or other pain management specialists that routinely provide inpatient services, although the pain and palliative care team refers patients to anesthesiology when interventional pain management procedures are required. The pain and palliative care service in this study provides care to patients with acute pain, patients with chronic pain experiencing acute exacerbations or intercurrent medical problems requiring hospitalization, and patients who are seriously or terminally ill and require palliative care including pain management. Many community hospital palliative care programs will be called on 
to manage both acute cancer pain and chronic orthopedic or neuropathic pain among noncancer patients. While the patientcentered, interdisciplinary team approach of a pain and palliative care team might be expected to improve pain outcomes in these noncancer patients, more research is needed in this area.

The large sample size and use of data from an established and well-utilized pain and palliative care department are strengths of this study. The data were consistently and prospectively collected in a standard fashion at the time of patient care. This study addresses an important area in palliative care in a population that is diverse and understudied.

There are several limitations of this study. This study examined patients receiving inpatient palliative care team consultations, and excluded patients receiving consultation as outpatients because of the initial/final pain outcome study design. The short time period of hospitalization for many patients, particularly those with more chronic types of pain, may have limited the ability to demonstrate pain reduction in these patients, and perhaps might be further explored in studies of patients receiving palliative care consultations as outpatients. The use of the 4-point affective verbal (none, mild, moderate, severe) rating is a limitation of the study, yet the 11-point pain scores in this study were collapsed to the 4point rating because many patients preferred to describe their pain in that manner, and one study goal was to include as broad a population as possible. Future areas of research should include examining pain reporting and measurement among the races in this study. Other patient characteristics which may affect pain measurement or reporting, such as socio-economic status, language barriers, health literacy levels, chronic pain, functional status, depression, and race of clinicians, were not examined, and are also important areas for future study.

Physician biases in referral to palliative care in terms of diagnoses and patient characteristics are important areas of future research. This study may have had a referral bias in which hospital patients were referred to the palliative care team. There were clearly more white patients with surgical diagnoses than with cancer or non-cancer medical diagnoses and more Asian and Pacific Islander patients with cancer and non-cancer medical diagnoses than surgical diagnoses. Surgical diagnoses posed a challenge for effective pain reduction with palliative care consultation, controlling for race. In contrast, after controlling for diagnosis, there were similar or improved pain outcomes with consultation among non-white patients compared to white patients. Further research is needed to determine the role of palliative care consultations in the optimal management of noncancer surgical pain, and in preventing disparities in pain management.

\section{Conclusions}

Patients demonstrated significant reductions in pain after inpatient pain and palliative care consultation. While more Hawaiian and Pacific Islander patients had pain reduction and less severe final pain scores than white patients, pain outcome differences associated with diagnoses were also significant. Palliative care consultations may serve as a powerful intervention to reduce disparities in pain management. Future work is needed to examine referral patterns in diverse populations and to enhance the management of non-cancer pain by palliative care teams.

\section{Acknowledgments}

This research was supported by: The John A. Hartford Foundation Center of Excellence in Geriatrics, University of Hawaii; Research Centers in Minority Institutions award, 1 R25 RR019321, from the National Center for Research Resources, National Institution of Health.

The authors would like to acknowledge James Davis, $\mathrm{PhD}$ for his assistance with statistical analyses, Kamal Masaki, MD for her assistance with study design, and Aida Wen, MD for her thoughtful review of the manuscript.

An abstract of this work was presented at the 2010 American Academy of Hospice and Palliative Medicine Annual Conference, Boston, Massachusetts, March 2010.

\section{Author Disclosure Statement}

No competing financial interests exist.

\section{References}

1. Lyon F, Boyd R, Mackway-Jones K: The convergent validity of the Manchester Pain Scale. Emerg Nurs 2005;13:34-38.

2. Cintron A, Morrison RS: Pain and ethnicity in the United States: A systematic review. J Palliat Med 2006;9:1454-1473.

3. Anderson KO, Green CR, Payne R: Racial and ethnic disparities in pain: Causes and consequences of unequal care. J Pain 2009;10:1187-1204.

4. Green CR, Montague L, Hart-Johnson TA: Consistent and breakthrough pain in diverse advanced cancer patients: A longitudinal examination. J Pain Symptom Manage 2009;37:831-847.

5. Pletcher MJ, Kertesz SG, Kohn MA, Gonzales R: Trends in opioid prescribing by race/ethnicity for patients seeking care in US emergency departments. JAMA 2008;299:70-78.

6. National Consensus Project for Quality Palliative Care. Clinical Practice Guidelines for Quality Palliative Care, Second Edition. www.nationalconsensusproject.org (Last accessed September 21, 2010).

7. Dhillon N, Kopetz S, Pei BL, Fabbro ED, Zhang T, Bruera E: Clinical findings of a palliative care consultation team at a comprehensive cancer center. J Palliat Med 2008;11:191-197.

8. Ngo-Metzger Q, Phillips RS, McCarthy EP: Ethnic disparities in hospice use among Asian-American and Pacific Islander patients dying with cancer. J Am Geriatr Soc 2008;56:139-144.

9. Cohen LL. Racial/ethnic disparities in hospice care: A systematic review. J Palliat Med 2008;11:763-768.

10. Edrington J, Sun A, Wong C, Dodd M, Padilla G, Paul S, Miaskowski C: Barriers to pain management in a community sample of Chinese American patients with cancer. J Pain Symptom Manage 2009;37:665-675.

11. Fernandes R, Braun KL, Ozawa J, Compton M, Guzman C, Somogyi-Zalud E: Home-based palliative care services for underserved populations. J Palliat Med 2010;13:413-419.

12. Im EO, Chee W, Guevara E, Liu Y, Lim HJ, Tsai HM, Clark M, Bender M, Suk Kim K, Hee Kim Y, Shin H: Gender and ethnic differences in cancer pain experience: A multiethnic survey in the United States. Nurs Res 2007;56:296-306.

13. Briggs $M$, Closs JS: A descriptive study of the use of visual analogue scales and verbal rating scales for the assessment of postoperative pain in orthopedic patients. J Pain Symptom Manage 1999;18:438-446.

14. Bruera E, Kuehn N, Miller MJ, Selmser P, Macmillan K: The Edmonton Symptom Assessment System (ESAS): A simple 
method for the assessment of palliative care patients. J Palliat Care 1991;7:6-9.

15. Williamson A, Hoggart B: Pain: A review of three commonly used pain rating scales. J Clin Nurs 2005;14:798-804.

16. Radbruch L, Sabatowski R, Loick G, Jonen-Thielemann I, Kasper M, Gondek B, Lehmann KA, Thielemann I: Cognitive impairment and its influence on pain and symptom assessment in a palliative care unit: Development of a Minimal Documentation System. Palliat Med 2000;14:266-276.

17. Selby D, Cascella A, Gardiner K, Do R, Moravan V, Myers J, Chow E: A single set of numerical cutpoints to define moderate and severe symptoms for the Edmonton Symptom Assessment System. J Pain Symptom Manage 2010;39:241-249.

18. Serlin RC, Mendoza TR, Nakamura Y, Edwards KR, Cleeland CS: When is cancer pain mild, moderate or severe? Grading pain severity by its interference with function. Pain 1995;61:277-284.

19. Feldt KS: The checklist of nonverbal pain indicators (CNPI). Pain Manag Nurs 2000;1:13-21.

20. NIH policy on reporting race and ethnicity data: Subjects in clinical research. http://grants.nih.gov/grants/guide/ notice-files/not-od-01-053.html] (Last accessed May 17, 2011).

21. Bell C, Kuriya M, Fischberg D: Hospice referrals and code status-Outcomes of inpatient palliative care consultations among Asian Americans and Pacific Islanders with cancer. J Pain Symptom Manage. (in press).

22. Cassell EJ, Leon AC, Kaufman SG: Preliminary evidence of impaired thinking in sick patients. Ann Intern Med 2001;134:1120-1123.

23. Morrison RS, Wallenstein S, Natale DK, Senzel RS, Huang LL: "We don't carry that"-Failure of pharmacies in predominantly nonwhite neighborhoods to stock opioid analgesics. N Engl J Med 2000;342:1023-1026.

24. Bonham VL: Race, ethnicity, and pain treatment: Striving to understand the causes and solutions to the disparities in pain treatment. J Law Med Ethics 2001;29:52-68.

25. Portenoy RK, Ugarte C, Fuller I, Haas G: Population-based survey of pain in the United States: Differences among white, African American, and Hispanic subjects. J Pain 2004;5:317-328.

26. Greenwald HP: Interethnic differences in pain perception. Pain 1991;44:157-163.

27. Hobara M: Beliefs about appropriate pain behavior: Crosscultural and sex differences between Japanese and EuroAmericans. Eur J Pain 2005;9:389-393.

28. Faucett J, Gordon N, Levine J: Differences in postoperative pain severity among four ethnic groups. J Pain Symptom Manage 1994;9:383-389.
29. Rabow MW, Dibble SL: Ethnic differences in pain among outpatients with terminal and end-stage chronic illness. Pain Med 2005;6:235-241.

30. Ware LJ, Epps CD, Herr K, Packard A: Evaluation of the Revised Faces Pain Scale, Verbal Descriptor Scale, Numeric Rating Scale, and Iowa Pain Thermometer in older minority adults. Pain Manag Nurs 2006;7:117-125.

31. Ezenwa MO, Ameringer S, Ward SE, Serlin RC: Racial and ethnic disparities in pain management in the United States. J Nurs Scholarsh 2006;38:225-233.

32. Gordon DB, Dahl JL, Miaskowski C, McCarberg B, Todd KH, Paice JA, Lipman AG, Bookbinder M, Sanders SH, Turk DC, Carr DB: American pain society recommendations for improving the quality of acute and cancer pain management: American Pain Society Quality of Care Task Force. Arch Intern Med 2005;165:1574-1580.

33. NCCN Clinical Practice Guidelines in Oncology-Cancer Pain 2010. www.nccn.org/professionals/physician_gls/ f_guidelines.asp (Last accessed August 21, 2011).

34. Cancer Pain Relief and Palliative Care. In: Report of World Health Organization Expert Committee Technical Report Series No. 804. World Health Organization: Geneva, Switzerland. http://www.who.int/cancer/publications/en (Last accessed March 15, 2011).

35. Tan EC, Lim Y, Teo YY, Goh R, Law HY, Sia AT: Ethnic differences in pain perception and patient-controlled analgesia usage for postoperative pain. J Pain 2008;9:849-855.

36. Manfredi PL, Morrison RS, Morris J, Goldhirsch SL, Carter JM, Meier DE: Palliative care consultations: How do they impact the care of hospitalized patients? J Pain Symptom Manage 2000;20:166-173.

37. O'Mahony S, Blank AE, Zallman L, Selwyn PA: The benefits of a hospital-based inpatient palliative care consultation service: Preliminary outcome data. J Palliat Med 2005;8:1033-1039.

38. Braiteh F, El Osta B, Palmer JL, Reddy SK, Bruera E: Characteristics, findings, and outcomes of palliative care inpatient consultations at a comprehensive cancer center. J Palliat Med 2007;10:948-955.

39. Jenkins CA, Schulz M, Hanson J, Bruera E: Demographic, symptom, and medication profiles of cancer patients seen by a palliative care consult team in a tertiary referral hospital. J Pain Symptom Manage 2000;19:174-184.

Address correspondence to: Christina L. Bell, M.D., M.Sc. 347 N. Kuakini Street HPM 9 Honolulu HI 96817

E-mail: bellcl@hawaii.edu 